\title{
THE RADIUS OF VANISHING BUBBLES IN EQUIVARIANT HARMONIC MAP FLOW FROM $D^{2}$ TO $S^{2 *}$
}

\author{
S. B. ANGENENT ${ }^{\dagger}$, J. HULSHOF ${ }^{\ddagger}$, AND H. MATANO $^{\S}$
}

Abstract. We derive an upper bound for the radius $R(t)$ of a vanishing bubble in a family of equivariant maps $F_{t}: D^{2} \rightarrow S^{2}$ which evolve by the harmonic map flow. The self-similar "type 1" radius would be $R(t)=C \sqrt{T-t}$. We prove that $R(t)=o(T-t)$.

Key words. harmonic map flow, asymptotics, singularities

AMS subject classifications. 35K55, 53C44

DOI. $10.1137 / 070706732$

1. Introduction. Let $N^{n} \subset \mathbb{R}^{k}$ be a smooth submanifold. The Dirichlet integral or energy of a map $F$ from the unit disc $D^{2} \subset \mathbb{R}^{2}$ into $N$ is defined to be

$$
\mathfrak{D}[F]=\frac{1}{2} \int_{D^{2}}|\nabla F(x)|^{2} .
$$

Extremals of this energy with prescribed boundary values $\left.F\right|_{\partial D^{2}}$ are called harmonic maps. Eells and Sampson [4] introduced the gradient flow for $\mathfrak{D}[F]$, now called the harmonic map flow, in which a family of maps $F_{t}: D \rightarrow N$ evolves according to the nonlinear heat equation

$$
\frac{\partial F}{\partial t}=(\Delta F)^{\top}
$$

Here, for any point $p \in N$ and vector $v \in T_{p} \mathbb{R}^{k}$, we write $v^{\top}$ for the tangential component of $v$ to $T_{p} N$.

When the target $N$ is the two-dimensional sphere, the harmonic map flow has recently appeared as a model for the direction field of a nematic liquid crystal; see [12] where the motivation comes from applications in fiber spinning, but physical applications go back as far as the treatment of ferromagnetic materials by Landau and Lifschitz [5].

For general targets (1.1) has been used in a purely mathematical context to construct harmonic maps of a given homotopy type; see, e.g., [7]. As a nonlinear vectorvalued partial differential equation, the harmonic map flow is of interest because of the possible formation of singularities, due to the presence of topological obstructions.

For targets with negative sectional curvatures Eells and Sampson showed that the initial value problem for (1.1) has a unique global solution $\left\{F_{t} \mid t \geq 0\right\}$, which

* Received by the editors October 29, 2007; accepted for publication (in revised form) April 16, 2009; published electronically July 30, 2009.

http://www.siam.org/journals/sima/41-3/70673.html

$\dagger$ Mathematics Department, UW Madison, Madison, WI (angenent@math.wisc.edu). Supported by NSF grant DMS-0101124 and a research visiting grant of NWO with additional support from the NDNS+ NWO research cluster and the Department of Mathematics of the Vrije Universiteit Amsterdam.

‡Afdeling Wiskunde, FEW, Vrije Universiteit Amsterdam, Amsterdam, The Netherlands (jhulshof@few.vu.nl).

$\S$ Graduate School of Mathematical Sciences, University of Tokyo, Komaba, Tokyo 153-8914, Japan (matano@ms.u-tokyo.ac.jp). 
converges to a harmonic map as $t \nearrow \infty$. Struwe $[8,6]$ later constructed global solutions for arbitrary targets $N$, which he allowed to have singularities at a finite number of points in space-time $D \times[0, \infty)$. That such singularities cannot be avoided was shown by examples of Chang, Ding, and Ye [2] as well as Coron and Ghidaglia [3]. For a nice treatment of their appearance and possible disappearance in the case of $N=S^{2}$ with radial symmetry, see [1].

Based on work of Struwe, Ding, Qing, Tian, Topping, and others (see Topping's papers $[10,9]$ and the references therein), one can give a good qualitative description of Struwe's solutions near their singular points. This description implies that whenever a singularity occurs a harmonic map $f: S^{2} \rightarrow N$ "bubbles off," i.e., for a singular point $(a, T) \in D \times(0, \infty)$ there exist times $t_{i} \nearrow T$, points $a_{i} \rightarrow a$, and scales $R_{i} \searrow 0$, as well as a nonconstant harmonic map $f: S^{2} \rightarrow N$ such that

$$
F_{t_{i}}\left(a_{i}+R_{i} x\right) \rightarrow f \circ \sigma(x) \quad(i \rightarrow \infty)
$$

uniformly in $x$ on compact subsets of $\mathbb{R}^{2}$. Here $\sigma: \mathbb{R}^{2} \rightarrow S^{2} \backslash\{p\}$ is the inverse of stereographic projection from the point $p \in S^{2}$. A full description involves the combination and/or superposition of several such "bubbles" (see $[9,10]$ ).

One can now ask at what rate the bubbles vanish, i.e., how large are the scales $R_{i}$ relative to the time to blow-up $T-t_{i}$ ? The natural scale, suggested by the parabolic equation, would be $R_{i}^{2} \approx C\left(T-t_{i}\right)$, but this can be ruled out. In fact, Topping [10] has shown that one always has

$$
R_{i}^{2}=o\left(\frac{T-t_{i}}{\left|\ln \left(T-t_{i}\right)\right|}\right),
$$

along some sequence $t_{i} \nearrow T$, while he also constructed a compact $C^{\infty}$ smooth target manifold $N$ and a solution $F: D \times[0, T) \rightarrow N$ with

$$
\liminf _{i \rightarrow \infty} R_{i}^{2}\left(T-t_{i}\right)^{-1-\delta}>0
$$

for any $\delta>0$, thus showing that the upper bound (1.2) cannot be improved in general.

In this note we consider the special case where the target $N$ is the perfectly round two-sphere $S^{2} \subset \mathbb{R}^{3}$ and where the maps $F^{t}: D^{2} \rightarrow S^{2}$ have rotational symmetry, i.e., the case studied in $[2,3,1]$. A later detailed analysis using formal matched asymptotic expansions by van den Berg, Hulshof, and King [11] strongly suggests that a variety of blow-up rates are possible, depending on the specified initial and boundary data. None of the formal solutions in [11] satisfy Topping's lower bound (1.3). In fact, the "generic case" in [11] has

$$
R(t) \sim \kappa \frac{T-t}{(\ln (T-t))^{2}}=o(T-t)
$$

for some constant $\kappa>0$ which varies from solution to solution.

Our main result here is a rigorous example of a solution to harmonic map flow for which we can give an upper bound for the blow-up rate of the radii $R_{i}$.

THEOREM 1.1. There exist a solution $F: D \times[0, T) \rightarrow S^{2}$ which forms a singularity at the origin at time $T$ and a decreasing function $R:[0, T) \rightarrow \mathbb{R}_{+}$such that

$$
\lim _{t \nearrow T} F^{t}(R(t) x)=\sigma(x)
$$


uniformly on compact subsets of $\mathbb{R}^{2}$. The length scale $R$ satisfies

$$
R(t)=o(T-t) \quad(t \nearrow T)
$$

and also an integrated version of this estimate

$$
\int_{0}^{T} \frac{R(t) d t}{(T-t)^{2}}<\infty
$$

In fact, we will derive the estimate for any solution $F$ whose initial data satisfies a certain monotonicity condition $(2.5 \mathrm{a}),(2.5 \mathrm{~b})$. In section 5 we note that such initial data are easily constructed.

Note that our upper estimates for the length scale $R(t)$ is less than Topping's generally valid estimate $(1.2)$ by a factor $\left(T-t_{i}\right)^{1 / 2+o(1)}$, while it differs only from the formal asymptotics (1.4) by logarithmic factors. This raises the question of which of the two behaviors (1.2) and (1.4) is more common: is the blow-up rate (1.4) exceptional and possible only in situations with a high degree of symmetry, or do most singular solutions of harmonic map flow blow up according to (1.4)?

Outline of the paper. We begin by describing the class of symmetric initial data we consider, and recall from the general theory that they do indeed produce solutions with finite time singularities. We establish a number of monotonicity properties of the solutions. Then, using the Sturmian theorem on intersections of solutions to parabolic equations in one dimension, we show that a bubble forms as $t \nearrow T$. This proof also gives us a quantitative estimate (Lemma 6.2 in section 6) on how close the singular bubble at time $t$ is to an actual harmonic map, and this leads to a weaker form of the lower bound for $R(t)$ in the theorem. In the end a careful analysis of the parabolic blow-up of the solution allows us to improve this estimate to $R(t)=o(T-t)$.

2. A class of solutions with symmetry. We describe here the class of solutions to which our estimates apply.

Rotational symmetry. When the target manifold is $N=S^{2} \subset \mathbb{R}^{3}$, the normal component of $\Delta F$ is $-|\nabla F|^{2} F$ so that (1.1) becomes

$$
\frac{\partial F_{t}}{\partial t}=\Delta F_{t}+\left|\nabla F_{t}\right|^{2} F_{t}
$$

We choose spherical coordinates $(\theta, \varphi)$ on $S^{2}$ and consider maps of the form

$$
F_{t}(r, \theta)=(\cos \theta \sin \varphi(r, t), \sin \theta \sin \varphi(r, t), \cos \varphi(r, t)) .
$$

Direct computation shows that harmonic map flow (2.1) preserves this class of maps and is equivalent to the following $\operatorname{PDE}$ for $\varphi$ :

$$
\frac{\partial \varphi}{\partial t}=\frac{\partial^{2} \varphi}{\partial r^{2}}+\frac{1}{r} \frac{\partial \varphi}{\partial r}-\frac{\sin 2 \varphi}{2 r^{2}}
$$

where $0 \leq r \leq 1$.

For $\varphi$ close to zero the last nonlinear term in (2.3) may be approximated by the linear term $-\varphi / r^{2}$. The resulting linear equation has a singularity in $r=0$, which forces bounded solutions to have a first order zero in $r=0$. This property of bounded solutions will result in the boundary condition at $r=0$ for $\varphi$ below. 


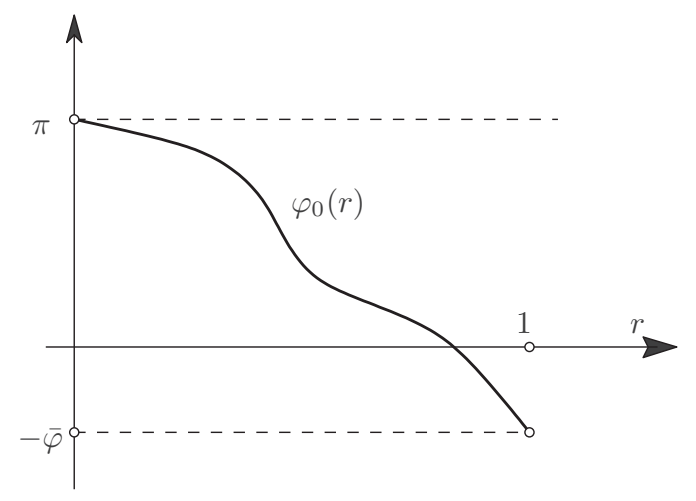

FIG. 2.1. The initial data.

The singularity at $r=0$ in (2.3) is caused by the use of spherical coordinates in the target and polar coordinates in the domain. When we approximate $\sin \phi$ by $\phi$, we replace the target $S^{2}$ by its tangent plane, described in polar coordinates with $\phi$ (or $\phi-k \pi$ for some integer $k$ ) acting as radius and $\theta$ as angular coordinate. All bounded solutions of (2.3) will have the property that for some $k$ the function $\phi(r, t)-k \pi$ has a first order zero in $r=0$. The solution wants to preserve this value of $k$. The singularities we are concerned with in this paper are forced to occur when this is no longer possible.

Next we choose initial and boundary conditions for $\varphi$ ensuring the occurence of a singularity. Choose some $0<\bar{\varphi}<\pi$ and consider harmonic map flows given by (2.2), where $\varphi:[0,1] \times[0, T)$ satisfies

$$
\varphi(1, t)=-\bar{\varphi}, \quad \varphi(0, t)=\pi .
$$

We shall assume that at time $t=0$ one has

$$
\begin{gathered}
\varphi(r, 0) \leq \pi, \\
\varphi_{r r}+\frac{1}{r} \varphi_{r}-\frac{\sin 2 \varphi}{2 r^{2}}<0
\end{gathered}
$$

for all $r \in[0,1]$.

Henceforth, $\varphi:[0,1] \times[0, T) \rightarrow \mathbb{R}$ denotes the corresponding maximal classical solution to (2.3) with boundary conditions $(2.4)$.

In section 5 we show that initial data $\varphi(r, 0)$ satisfying the hypotheses $(2.5 \mathrm{a})$ and (2.5b) actually do exist (see Figure 2.1). It follows from the work of Chang, Ding, and Ye [2] that any solution whose initial data satisfy (2.5a) and (2.5b) will indeed become singular in finite time.

Monotonicity properties. In section 3 we will use the maximum principle to prove the following.

LEMMA 2.1. $\varphi_{t}(r, t)<0$ for all $(r, t) \in(0,1) \times[0, T)$.

One could try to use the maximum principle to show that $\varphi_{r}<0$ is also preserved by the flow. However, this turns out to be a consequence of the condition $\varphi_{t}<0$, which we have imposed on our initial data.

LEMMA 2.2. $\varphi_{r}(r, t)<0$ for all $(r, t) \in(0,1) \times[0, T)$.

See section 5 for the proof. 
The radius of the bubble. Because of (2.4) and $\varphi_{r}<0$, there is a unique $R(t) \in(0,1)$ for each $t \in[0, T)$ such that

$$
\varphi(R(t), t)=\pi / 2,
$$

i.e., the corresponding map $F^{t}$ maps the circle in $D^{2}$ with radius $R(t)$ to the equator in $S^{2}$. By the implicit function theorem, $R(t)$ is a monotonically decreasing function of time with

$$
R^{\prime}(t)=-\varphi_{t}(R(t), t) / \varphi_{r}(R(t), t) .
$$

The radius $R(t)$ defined here is the one we meant in Theorem 1.1.

For any initial function $\varphi_{0}$ satisfying our hypotheses $(2.5 \mathrm{a}),(2.5 \mathrm{~b})$ there exist small $\varepsilon>0$ and large $T_{*}>0$ such that the Chang, Ding, and Ye supersolution $\Phi^{\varepsilon, T_{*}}(\cdot, 0)$ lies above $\varphi_{0}$ at $t=0$. By the maximum principle, this continues to hold for $t>0$, and, as argued in [2], the solution $\varphi$ must become singular before $t=T_{*}$.

Suppose that the solution becomes singular at time $T<T_{*}$; then along some sequence of times $t_{i} \nearrow T$ and points $p_{i} \in D^{2}$, a blow-up of the maps $F^{t}$ will result in a nontrivial harmonic map from $\mathbb{R}^{2} \rightarrow S^{2}$. The limit map will inherit the symmetries of the maps $F^{t_{i}}$. Because of this, the only possible blow-up point is the origin, and the only possible blow-up map is inverse stereographic projection. We therefore conclude from the general theory that along some sequence $t_{i} \nearrow T$ one has $R\left(t_{i}\right) \searrow 0$, and

$$
\lim _{i \rightarrow \infty} \varphi\left(R\left(t_{i}\right) z, t_{i}\right)=\pi-2 \arctan z .
$$

Since the bubble radius $R(t)$ is a monotone function of time, we immediately have the following stronger statement.

LEMma 2.3. The maximal classical solution $\varphi$ becomes singular in finite time, i.e., $T<\infty$. Moreover, $\lim _{t \nearrow T} R(t)=0$.

In Lemma 6.2 we will show that the bubble forms for all $t$ close to $T$ instead of just along a sequence $t_{i} \nearrow T$.

Lemma 2.4. One has

$$
\lim _{t \nearrow T} \varphi(R(t) z, t)=\pi-2 \arctan z
$$

uniformly on bounded $z$ intervals.

3. Proof of Lemma 2.1. It will be convenient to abbreviate

$$
f(\varphi)=\frac{1}{2} \sin 2 \varphi=\sin \varphi \cos \varphi
$$

We consider $u=\varphi_{t}$ and $v=u(r, t) / r$. For $u$ one computes

$$
u_{t}=u_{r r}+\frac{1}{r} u_{r}-\frac{f^{\prime}(\varphi(r, t))}{r^{2}} u \text {. }
$$

From this one obtains

$$
v_{t}=v_{r r}+\frac{3}{r} v_{r}+\frac{1-f^{\prime}(\varphi(r, t))}{r^{2}} v .
$$

Since $\varphi$ comes from a classical solution of harmonic map flow, we have

$$
\left|\varphi_{t}(r, t)\right|=\left|\partial_{t} F^{t}(r, \theta)\right|,
$$


where the right-hand side actually does not depend on $\theta$. For arbitrary $\delta>0$ the map $F^{t}$ is smooth on $D^{2} \times[0, T-\delta]$, so we have $\left|\partial_{t} F^{t}\right| \leq C r$ (the constant $C$ may depend on $\delta$ ). Consequently, $v(r, t)=\varphi_{t}(r, t) / r$ is uniformly bounded for $0<r<1$, $0 \leq t \leq T-\delta$.

We also may conclude from the smoothness of $F_{t}$, i.e. from the boundedness of $\left|\nabla F_{t}\right|$, that

$$
|\varphi(r, t)-\pi| \leq C_{\delta} r \quad \text { for } \quad 0<r<1, \quad 0 \leq t \leq T-\delta
$$

and, hence,

$$
\left|1-f^{\prime}(\varphi(r, t))\right|=|1-\cos \varphi(r, t)| \leq \varphi^{2} \leq C_{\delta}^{2} r^{2}
$$

Thus $v$ satisfies

$$
v_{t}=v_{r r}+\frac{3}{r} v_{r}+Q(r, t) v
$$

where $Q(r, t)=r^{-2}\left(1-f^{\prime}(\varphi(r, t))\right)$ is uniformly bounded.

The differential operator in (3.1) is the radial Laplacian in $\mathbb{R}^{4}$ with a bounded potential added, so the weak maximum principle holds. Therefore, $v(r, 0)<0$ (assumption (2.5b)) implies $v(r, t) \leq 0$ for $0 \leq r \leq 1$ and $0<t<T$. The strong maximum principle then implies $v(r, t)<0$ and hence $\varphi_{t}<0$ for $0<r<1$ and $0<t<T$.

4. The $\boldsymbol{x}$ variable and the energy $\boldsymbol{E}$. Instead of considering (2.3) we change the independent variable $r$ to $x=-\ln r$ (so $0<r<1$ implies $x>0$ ) and study the PDE

$$
\varphi_{t}=e^{2 x}\left(\varphi_{x x}-f(\varphi)\right)
$$

in the domain $0<x<\infty, 0 \leq t<T$, with boundary conditions

$$
\varphi(0, t)=-\bar{\varphi}, \quad \varphi(\infty, t)=\pi .
$$

Time independent solutions of (4.1) satisfy the ODE

$$
\varphi^{\prime \prime}=f(\varphi),
$$

where $f(\varphi)=\frac{1}{2} \sin 2 \varphi$. This equation has

$$
E=\frac{1}{2} \varphi^{\prime}(x)^{2}-\frac{1}{2} \sin ^{2} \varphi(x)
$$

as first integral. It follows that there is exactly one solution $\varphi(E, x)$ of $(4.3)$ which has $\varphi(0)=\frac{1}{2} \pi$ and whose "energy" is $E$. This solution is determined by the relation

$$
-x=\int_{\varphi(E, x)}^{\pi / 2} \frac{\mathrm{d} \varphi}{\sqrt{2 E+\sin ^{2} \varphi}} .
$$

For $E=0$ this leads to the unique solution $\Phi(x)$, with $\Phi(-\infty)=0, \Phi(+\infty)=\pi$, and $\Phi(0)=\pi / 2$, which corresponds to stereographic projection, namely,

$$
\Phi(x)=2 \arctan e^{x} .
$$




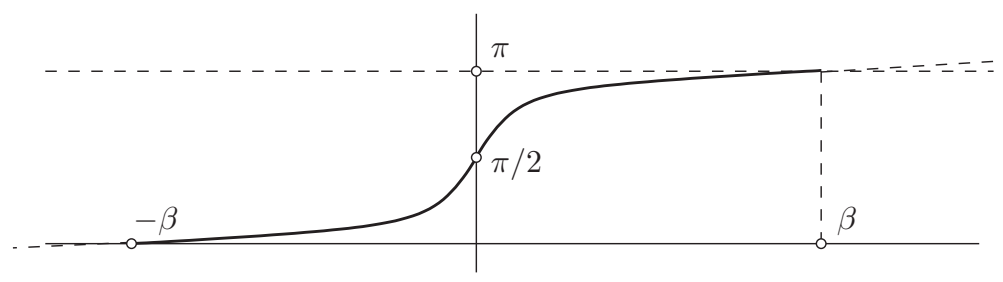

FIG. 4.1. $\Phi_{\beta}$.

When $E<0$, one is led to periodic solutions $\varphi$, which we shall not need in this paper. For each $E>0$ we set

$$
\beta(E)=\int_{0}^{\pi / 2} \frac{\mathrm{d} \varphi}{\sqrt{2 E+\sin ^{2} \varphi}}
$$

so that the solution with energy $E$ satisfies

$$
\varphi(-\beta)=0, \quad \varphi(0)=\pi / 2, \quad \varphi(\beta)=\pi .
$$

See Figure 4.1.

Clearly, $\beta(E)$ is a monotone function of $E$ with $\beta(E) \rightarrow \infty$ as $E \rightarrow 0$. We denote the inverse by $E=E_{\beta}$, and we write $\Phi_{\beta}(x)=\varphi\left(E_{\beta}, x\right)$. The function $\Phi_{\beta}$ is thus the unique solution of (4.3) which satisfies the boundary conditions (4.7). One has

$$
\lim _{\beta \rightarrow \infty} \Phi_{\beta}(x)=\Phi(x) .
$$

Lemma 4.1. The energy $E_{\beta}$ of $\Phi_{\beta}$ satisfies

$$
E_{\beta}=8 e^{-2 \beta+o(1)} \quad(\beta \rightarrow \infty) .
$$

Proof. We have

$$
\begin{aligned}
\beta & =\int_{0}^{\pi / 2} \frac{d \varphi}{\sqrt{2 E+\sin ^{2} \varphi}} \\
& =\int_{0}^{\pi / 2} \frac{1-\cos \varphi}{\sqrt{2 E+\sin ^{2} \varphi}} d \varphi+\int_{0}^{\pi / 2} \frac{\cos \varphi}{\sqrt{2 E+\sin ^{2} \varphi}} d \varphi \\
& =A+B .
\end{aligned}
$$

In the first term we may simply let $E$ tend to 0 because of monotone convergence. One gets

$$
\lim _{E \searrow 0} A=\int_{0}^{\pi / 2} \frac{1-\cos \varphi}{\sin \varphi} d \varphi=\ln 2 .
$$

For the second term one finds

$$
B=\left[\operatorname{arsinh} \frac{\sin \varphi}{\sqrt{2 E}}\right]_{0}^{\pi / 2}=\operatorname{arsinh} \frac{1}{\sqrt{2 E}} .
$$

Adding $A$ and $B$ while using $\operatorname{arsinh} t=\ln \left(t+\sqrt{1+t^{2}}\right)=\ln 2 t+\frac{1}{4} t^{-2}+\cdots$ for $t \rightarrow \infty$, we arrive at

$$
\beta=\ln 2+o(1)+\operatorname{arsinh} \frac{1}{\sqrt{2 E}}=\frac{1}{2} \ln (8 / E)+o(1),
$$

from which (4.8) follows. 
Lemma 4.2. For all $\beta>0$ and all $x \geq 0$ one has

$$
0<\Phi_{\beta}(x)-\Phi(x)<E_{\beta} \sinh x \leq C e^{-2 \beta} \sinh x .
$$

Since $\Phi_{\beta}-\Phi$ is an odd function, one has the opposite inequalities for $x<0$.

Proof. From the construction one sees that $\Phi_{\beta}$ is a monotone function of $\beta$. This implies $\Phi_{\beta}>\Phi$.

Both $\Phi$ and $\Phi_{\beta}$ are solutions of the $\operatorname{ODE} \varphi^{\prime \prime}-f(\varphi)=0$, so their difference $z=\Phi_{\beta}-\Phi$ satisfies $z^{\prime \prime}-Q(x) z=0$, where, by the mean value theorem, one has

$$
|Q(x)|=\left|\frac{f\left(\Phi_{\beta}(x)\right)-f(\Phi(x))}{\Phi_{\beta}(x)-\Phi(x)}\right|<1 .
$$

This implies that $z^{\prime \prime}-z=(Q(x)-1) z<0$. Therefore, taking into account that $z(0)=0$, one finds

$$
\begin{aligned}
z(x) & =z(0) \cosh x+z^{\prime}(0) \sinh x+\int_{0}^{x} \sinh (x-\xi)\left\{z^{\prime \prime}(\xi)-z(\xi)\right\} d \xi \\
& <z^{\prime}(0) \sinh x
\end{aligned}
$$

for all $x>0$. Finally,

$$
z^{\prime}(0)=\Phi_{\beta}^{\prime}(0)-\Phi^{\prime}(0)=\frac{\Phi_{\beta}^{\prime}(0)^{2}-\Phi^{\prime}(0)^{2}}{\Phi_{\beta}^{\prime}(0)+\Phi^{\prime}(0)}<E_{\beta},
$$

because $\Phi_{\beta}^{\prime}(0)>\Phi^{\prime}(0)=1$.

5. Initial data and their intersections with steady states. Rewritten in the $x$ variable the hypotheses $(2.5 \mathrm{a})$ and $(2.5 \mathrm{~b})$ are

$$
\begin{gathered}
\varphi_{0}(x) \leq \pi \text { and } \varphi_{0}^{\prime \prime}-f\left(\varphi_{0}\right)<0 \text { for } 0 \leq x<\infty, \\
\lim _{x \rightarrow \infty} \varphi_{0}(x)=\pi .
\end{gathered}
$$

Lemma 5.1. Let $\varphi_{0}:[0, \infty) \rightarrow \mathbb{R}$ be a function which satisfies (5.1a) and (5.1b). Then $\varphi_{0}^{\prime}(x)>0$ for all $x \geq 0$.

As a consequence, Lemma 2.1 implies Lemma 2.2.

Proof. There must be a final interval $\left[x_{1}, \infty\right)$ on which $\frac{1}{2} \pi \leq \varphi_{0}(x) \leq \pi$. On this interval one has $\varphi_{0}^{\prime \prime}<f\left(\varphi_{0}\right) \leq 0$, so $\varphi_{0}$ is concave there. Hence for $x \geq x_{1}$ we already have $\varphi_{0}^{\prime}(x)>0$.

Let $x_{2} \geq 0$ be the largest root of $\varphi^{\prime}(x)=0$ if such exists. If we define $E_{\varphi_{0}}(x)=$ $\frac{1}{2}\left\{\varphi_{0}^{\prime}(x)^{2}-\sin ^{2} \varphi_{0}(x)\right\}$, then for $x>x_{2}$ we have

$$
\frac{d}{d x} E_{\varphi_{0}}(x)=\varphi_{0}^{\prime}(x)\left\{\varphi_{0}^{\prime \prime}-f\left(\varphi_{0}\right)\right\}<0 .
$$

Since $\lim _{x \rightarrow \infty} E_{\varphi_{0}}(x)=0$, we get $E_{\varphi_{0}}\left(x_{2}\right)>0$. On the other hand

$$
E_{\varphi_{0}}\left(x_{2}\right)=\frac{1}{2}\left\{\varphi_{0}^{\prime}\left(x_{2}\right)^{2}-\sin ^{2} \varphi_{0}\left(x_{2}\right)\right\}=-\frac{1}{2} \sin ^{2} \varphi_{0}\left(x_{2}\right) \leq 0,
$$

a contradiction.

Hence no such $x_{2}$ can exist, and we find that $\varphi_{0}^{\prime}(x)>0$ for all $x \geq 0$.

The proof also shows that $E_{\varphi_{0}}(x)$ is strictly decreasing for all $x \geq 0$ (by (5.2)) and hence that $E_{\varphi_{0}}(x)>0$ for all $x \geq 0$. 
Construction of the initial data. It follows from Lemma 5.1 that for any initial $\varphi_{0}$ which satisfies (5.1a), (5.1b) one can invert the map $x \mapsto \varphi_{0}(x)$ and thus construct a function $\mathcal{G}:[-\bar{\varphi}, \pi] \rightarrow[0, \infty)$ for which one has $\varphi_{0}^{\prime}(x)=\mathcal{G}\left(\varphi_{0}(x)\right)$. This function must satisfy $\mathcal{G}(\pi)=0$, of course, but also

$$
\frac{d}{d \phi}\left(\frac{1}{2} \mathcal{G}(\phi)^{2}-\frac{1}{2} \sin ^{2} \phi\right)<0 \quad \text { for } \quad-\bar{\varphi}<\phi<\pi,
$$

since, by the chain rule and in view of $\varphi_{0}^{\prime}(x)=\mathcal{G}\left(\varphi_{0}(x)\right)$, the left-hand side equals

$$
\frac{\frac{d}{d x}\left(\frac{1}{2} \varphi_{0}^{\prime}(x)^{2}-\frac{1}{2} \sin ^{2} \varphi_{0}(x)\right)}{\varphi_{0}^{\prime}(x)}=\varphi_{0}^{\prime \prime}(x)-f\left(\varphi_{0}(x)\right)<0
$$

when $\phi=\varphi_{0}(x)$.

Conversely, let $\mathcal{E}:[-\bar{\varphi}, \pi] \rightarrow[0, \infty)$ be any smooth decreasing function for which $\mathcal{E}(\pi)=0$, and define

$$
\mathcal{G}(\phi)=\sqrt{2\left(\mathcal{E}(\phi)+\sin ^{2} \phi\right)}
$$

Then $\mathcal{G}(\phi)$ satisfies (5.3). The solution $\varphi_{0}:[0, \infty) \rightarrow[-\bar{\varphi}, \pi)$ of

$$
\varphi^{\prime}(x)=\mathcal{G}(\varphi(x)), \quad \varphi(0)=-\bar{\varphi}
$$

is an increasing function with $\lim _{x \rightarrow \infty} \varphi_{0}(x)=\pi$. Moreover, (5.4) implies that $\varphi_{0}$ satisfies $\varphi_{0}^{\prime \prime}-f\left(\varphi_{0}\right)<0$ so that $\varphi_{0}$ satisfies our hypotheses (5.1a) and (5.1b). So, we have constructed an admissible initial value for each smooth decreasing function $\mathcal{E}:[-\bar{\varphi}, \pi] \rightarrow[0, \infty)$ with $\mathcal{E}(\pi)=0$.

Intersections. We now count intersections of $\varphi_{0}$ with steady states.

LEMMA 5.2. The graph of $\varphi_{0}(x)$ intersects the graph of $\Phi(x-\zeta)$ at most once (for any $\zeta \in \mathbb{R}$ ).

Proof. All $\Phi(x-\zeta)$ have zero energy, i.e., $E_{\Phi} \equiv 0$. If $\varphi_{0}\left(x_{1}\right)=\Phi\left(x_{1}-\zeta\right)$, then $E_{\varphi_{0}}\left(x_{1}\right)>E_{\Phi}\left(x_{1}-\zeta\right)=0$ implies $\varphi_{0}^{\prime}\left(x_{1}\right)>\Phi^{\prime}\left(x_{1}-\zeta\right)$. If there were more than one intersection, at least one of them would have to have $\varphi_{0}^{\prime}\left(x_{1}\right) \leq \Phi^{\prime}(x-\zeta)$.

Lemma 5.3. The graphs of $\varphi_{0}$ and $\Phi_{\beta}(x-\zeta)$ intersect at most twice for any $\zeta \in \mathbb{R}$.

Proof. If some point of intersection $x_{1} \geq 0$ has $\varphi_{0}^{\prime}\left(x_{1}\right) \leq \Phi_{\beta}^{\prime}\left(x_{1}-\zeta\right)$, then one has $E_{\varphi_{0}}\left(x_{1}\right) \leq E_{\Phi_{\beta}}\left(x_{1}\right)$. But $E_{\Phi_{\beta}}$ is constant and $E_{\varphi_{0}}$ decreases, so for all $x>x_{1}$ one has $E_{\varphi_{0}}(x)<E_{\Phi_{\beta}}(x)$. This implies that there cannot be any further intersections after $x=x_{1}$, for at such an intersection one would have $\varphi_{0}^{\prime}(x) \geq \Phi_{\beta}^{\prime}(x-\zeta)$ and thus $E_{\varphi_{0}}(x) \geq E_{\Phi_{\beta}}$.

Consequently, there cannot be more than two intersections. For if there were three intersections, say, at $x_{1}<x_{2}<x_{3}$, then at either $x_{1}$ or $x_{2}$ one would have $\varphi_{0}^{\prime} \leq \Phi_{\beta}^{\prime}$, and the third intersection at $x_{3}$ could not occur by the argument in the preceding paragraph.

6. Proof of Lemma 2.4 with an error estimate. In section 4 we showed that $\varphi_{t}<0$, i.e., $\varphi_{x x}-f(\varphi)<0$ at each time $t$. Hence each $\varphi(\cdot, t)$ satisfies the hypotheses of Lemma 2.2, and it follows that $\varphi_{x}>0$ for all $(x, t)$. In particular there exists a unique $X(t)$ such that $\varphi(X(t), t)=\frac{1}{2} \pi$. The radius $R(t)$ from (2.6) is given by $R(t)=e^{-X(t)}$. 


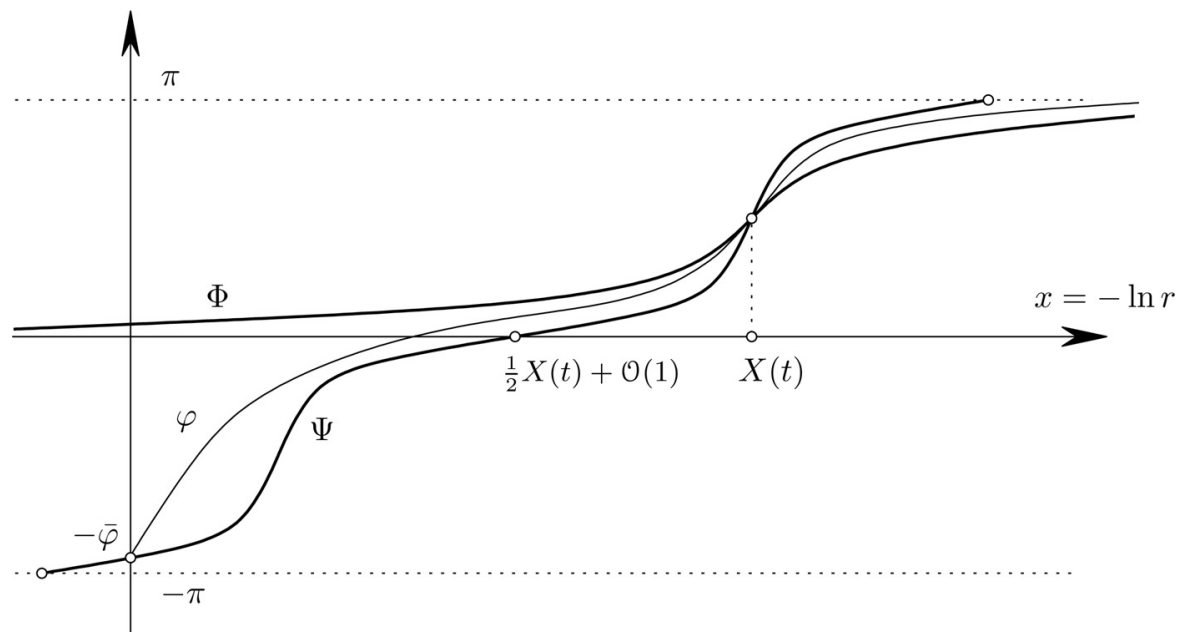

FIG. 6.1. The proof of Lemma 2.4.

Again, $\varphi_{x x}-f(\varphi)<0$ implies that $\varphi(\cdot, t)$ also satisfies Lemma 5.2 and hence the graphs of $\varphi(\cdot, t)$ and $\Phi(x-X(t))$ intersect only once: the intersection occurs by definition of $X(t)$ at $x=X(t)$. We conclude that

$$
\left\{\begin{array}{l}
\varphi(x, t)>\Phi(x-X(t)) \text { for } \quad x>X(t) \\
\varphi(x, t)<\Phi(x-X(t)) \text { for } \quad x<X(t) .
\end{array}\right.
$$

See Figure 6.1. Next, we compare $\varphi(\cdot, t)$ with $\Psi(x)$, where $\Psi(x)$ is the unique solution of

$$
\Psi^{\prime \prime}-f(\Psi)=0, \quad \Psi(X(t))=\frac{\pi}{2}, \quad \Psi(0)=-\bar{\varphi} .
$$

One can extend $\Psi(x)$ to a function on all $\mathbb{R}$ by solving the ODE $\Psi^{\prime \prime}=f(\Psi)$.

Lemma 6.1. $\Psi(x)=\Phi_{\beta}(x-X(t))$, where

$$
\beta=\frac{1}{2} X(t)+\mathcal{O}(1)
$$

Proof. Since $\Psi(x)$ crosses the $\varphi=0$ line, it must be a positive energy solution of the ODE and hence $\Psi(x)=\Phi_{\beta}(x-X(t))$ for some $\beta$. At $x=0$ one has $\Psi(0)=-\bar{\varphi}$, which is bounded away from 0 and $-\pi$, so within a distance ${ }^{1}$ of $\mathcal{O}(1)$ there must be a point $x_{1}$ with $\Psi\left(x_{1}\right)=-\frac{1}{2} \pi$. Clearly, $X(t)-x_{1}=2 \beta$.

Lemma 6.2. For all $x \geq 0$ one has

$$
|\varphi(x, t)-\Phi(x-X(t))| \leq C e^{-X(t)}|\sinh (x-X(t))| .
$$

Proof. By Lemma 5.3 the graphs of $\varphi(x, t)$ and $\Psi(x)$ intersect at most twice, which they do at $x=0$ and at $x=X(t)$. Hence we have

$$
\begin{cases}\varphi(x, t)<\Psi(x) & \text { for } x>X(t) \\ \varphi(x, t)>\Psi(x) & \text { for } x<X(t)\end{cases}
$$

\footnotetext{
${ }^{1}$ In fact, using $E_{\beta} \geq 0$ one can estimate $x_{1}$ by $\left|x_{1}\right|=\left|\int_{\frac{\varphi}{\varphi}}^{\pi / 2}\left(2 E_{\beta}+\sin ^{2} \varphi\right)^{-1 / 2} d \varphi\right| \leq$ $|\operatorname{artanh} \cos \bar{\varphi}|$.
} 
If we combine this with (6.1) we get

$$
|\varphi(x, t)-\Phi(x-X(t))| \leq|\Psi(x)-\Phi(x-X(t))|
$$

for all $x \geq 0$. Since $\Psi(x)=\Phi_{\beta}(x-X(t))$, the inequality (6.4) follows from Lemma 4.2 .

7. Convergence of higher derivatives. We are considering $\varphi$ as a function of $x=-\ln r$ and $t$ so that $\varphi$ satisfies (4.1), (4.2). In these variables blow-up of the harmonic map flow leads to unbounded time derivatives but not to unbounded space derivatives.

Lemma 7.1. For all $m=0,1,2, \ldots$, there are constants $M_{m, \delta}$ such that

$$
\left|\frac{\partial^{m} \varphi}{\partial x^{m}}(x, t)\right| \leq M_{m, \delta}
$$

holds for $x \geq 1$ and $t \geq \delta$.

Proof. Let $x_{0} \geq 1$ and $t_{0} \in[\delta, T)$ be given. Then consider

$$
\widetilde{\varphi}(x, t)=\varphi\left(x_{0}+x, t_{0}+e^{-2 x_{0}} t\right) .
$$

The function $\widetilde{\varphi}$ satisfies (4.1) on the rectangle $-1<x<1,-\delta<t \leq 0$ (in fact for $\left.-\delta e^{2 x_{0}}<t \leq 0\right)$ and is bounded there.

By standard interior estimates for semilinear parabolic equations, we now find that all derivatives $\partial_{x}^{m} \widetilde{\varphi}(0,0)$ are bounded. This implies (7.1).

Recall that for any integers $0 \leq k \leq m$ there is a constant $C_{k, m}$ such that any $C^{m}$ function on the interval $[-L, L]$ satisfies

$$
\left\|f^{(k)}\right\|_{\infty} \leq C_{k, m}\|f\|_{\infty}^{1-k / m}\left\|f^{(m)}\right\|_{\infty}^{k / m},
$$

$\|\cdots\|_{\infty}$ being the supremum norm on the interval $[-L, L]$. If we apply this interpolation inequality to (6.4) and (7.1), we find that $\varphi(X(t)+z, t)$ converges in $C^{\infty}$ to $\Phi(z)$. More precisely, we get the following estimates.

LEMma 7.2. For any $0 \leq k \leq m$ there is a constant $C_{k, m, L}$ such that

$$
\sup _{|x-X(t)| \leq L}\left|\frac{\partial^{k} \varphi}{\partial x^{k}}-\Phi^{(k)}\right| \leq C_{k, m, L} e^{-(1-k / m) X(t)} .
$$

8. The mollified logarithmic radius $\hat{\boldsymbol{X}}$. We consider the following alternative to $X(t)$ : define $\hat{X}(t)$ by requiring

$$
\int_{-1}^{+1} \eta(s) \varphi(\hat{X}(t)+s, t) d s=\frac{\pi}{2}
$$

where $0 \leq \eta \in C_{c}^{\infty}(-1,1)$ is some function with $\int_{-1}^{1} \eta(s) d s=1$. The left-hand side here is strictly increasing as a function of $\hat{X}(t)$ so that uniqueness of $\hat{X}(t)$ is ensured.

We also define the corresponding mollified radius

$$
\hat{R}(t)=e^{-\hat{X}(t)} .
$$

Lemma 8.1. $|\hat{X}(t)-X(t)| \leq C e^{-X(t)}$ for some $C<\infty$.

Consequently, one also has

$$
X(t)=\hat{X}(t)+o(1) \text { and } R(t)=(1+o(1)) \hat{R}(t) \text { as } t \nearrow T .
$$


Proof. It follows from Lemma 6.2 that

$$
\int_{-1}^{+1} \eta(s) \varphi(X(t)+s, t) d s=\frac{\pi}{2}+\mathcal{O}\left(e^{-X(t)}\right) .
$$

It also follows from Lemma 7.2 that

$$
A(\xi) \stackrel{\text { def }}{=} \int_{-1}^{+1} \eta(s) \varphi(X(t)+\xi+s, t) d s
$$

satisfies

$$
A^{\prime}(\xi)=\int_{-1}^{+1} \eta(s) \varphi_{x}(X(t)+\xi+s, t) d s \geq \delta>0
$$

for some constant $\delta$, and all $|\xi| \leq 1$.

Writing $\hat{X}(t)=X(t)+\xi$, these two inequalities imply the lemma.

We proceed to compute $\hat{X}^{\prime}(t)$. Differentiation of the defining relation for $\hat{X}(t)$ gives

$$
0=\frac{d}{d t} \int_{-1}^{1} \eta(s) \varphi(\hat{X}(t)+s, t) d t=\int_{-1}^{1} \eta(s)\left\{\varphi_{t}+\hat{X}^{\prime}(t) \varphi_{x}\right\} d s
$$

so that

$$
\hat{X}^{\prime}(t)=-\frac{\int_{-1}^{1} \eta(s) \varphi_{t}(\hat{X}(t)+s, t) d s}{\int_{-1}^{1} \eta(s) \varphi_{x}(\hat{X}(t)+s, t) d s} .
$$

It is immediately clear from $\varphi_{t}<0$ and $\varphi_{x}>0$ that

$$
\hat{X}^{\prime}(t)>0 \text {. }
$$

Moreover, the PDE (4.1) for $\varphi$ implies

$$
\hat{X}^{\prime}(t)=-\frac{\int_{-1}^{1} \eta(s) e^{2(\hat{X}+s)}\left(\varphi_{s s}-\frac{1}{2} \sin 2 \varphi\right) d s}{\int_{-1}^{1} \eta(s) \varphi_{s}(\hat{X}+s, t) d s} .
$$

(Note that for fixed $t$ one has $\partial / \partial x=\partial / \partial s$.) After factoring out the $e^{2 \hat{X}}$ and integrating by parts twice in the numerator and once in the denominator, one gets

$$
\hat{X}^{\prime}(t)=e^{2 \hat{X}(t)} \frac{\int_{-1}^{1}\left\{\left(\eta(s) e^{2 s}\right)_{s s} \varphi-\eta(s) e^{2 s} \frac{1}{2} \sin 2 \varphi\right\} d s}{\int_{-1}^{1} \eta^{\prime}(s) \varphi(\hat{X}+s, t) d s} .
$$

By Lemma 6.2, we find for the denominator

$$
\begin{aligned}
\int_{-1}^{1} \eta^{\prime}(s) \varphi(\hat{X}+s, t) d s & =\int_{-1}^{1} \eta^{\prime}(s) \Phi(s) d s+\mathcal{O}\left(e^{-\hat{X}(t)}\right) \\
& =-\int_{-1}^{1} \eta(s) \Phi^{\prime}(s) d s+\mathcal{O}\left(e^{-\hat{X}(t)}\right) \\
& =-C_{0}+\mathcal{O}\left(e^{-\hat{X}(t)}\right)
\end{aligned}
$$

in which $C_{0}$ is some positive constant. 
For the numerator we get, using Lemma 6.2 again,

$$
\begin{aligned}
\int_{-1}^{1} & \left\{\left(\eta(s) e^{2 s}\right)_{s s} \varphi-\eta(s) e^{2 s} \frac{1}{2} \sin 2 \varphi\right\} d s \\
& =\int_{-1}^{1}\left\{\left(\eta(s) e^{2 s}\right)_{s s} \Phi(s)-\eta(s) e^{2 s} \frac{1}{2} \sin 2 \Phi(s)\right\} d s+\mathcal{O}\left(e^{-\hat{X}(t)}\right) \\
& =\int_{-1}^{1} \eta(s) e^{2 s}\left\{\Phi^{\prime \prime}(s)-\frac{1}{2} \sin 2 \Phi(s)\right\} d s+\mathcal{O}\left(e^{-\hat{X}(t)}\right) \\
& =\mathcal{O}\left(e^{-\hat{X}(t)}\right)
\end{aligned}
$$

in which we have used that $\Phi$ satisfies the differential equation $\Phi^{\prime \prime}(s)=\frac{1}{2} \sin 2 \Phi(s)$.

These last two computations applied to (8.3) give us

$$
\hat{X}^{\prime}(t)=\mathcal{O}\left(e^{\hat{X}(t)}\right)
$$

which is the main estimate we derive in this section. Since $\hat{R}(t)=e^{-\hat{X}(t)}$, we have

$$
\frac{d \hat{R}}{d t}=-e^{-\hat{X}(t)} \hat{X}^{\prime}(t)=\mathcal{O}(1),
$$

which implies $\hat{R}(t)=\mathcal{O}(T-t)$ and, by Lemma 8.1,

$$
R(t)=\mathcal{O}(T-t) .
$$

9. $\boldsymbol{m}(\boldsymbol{\tau})$ and $\boldsymbol{Y}(\boldsymbol{\tau})$. We consider the parabolic blow-up of our solution to harmonic map flow. Let

$$
\begin{aligned}
& \varphi(r, t)=u\left(\frac{r}{\sqrt{T-t}},-\ln (T-t)\right), \\
& y=\frac{r}{\sqrt{T-t}}, \quad \tau=-\ln (T-t) .
\end{aligned}
$$

Then $u(y, \tau)$ is defined for $0 \leq y \leq e^{\tau / 2},-\ln T \leq \tau<\infty$, where it satisfies

$$
\begin{aligned}
u_{\tau} & =u_{y y}+\left(\frac{1}{y}-\frac{y}{2}\right) u_{y}-\frac{1}{y^{2}} u+\frac{g(u)}{y^{2}}, \\
& =\frac{1}{y} e^{y^{2} / 4}\left(y e^{-y^{2} / 4} u_{y}\right)_{y}-\frac{1}{y^{2}} u+\frac{g(u)}{y^{2}},
\end{aligned}
$$

in which

$$
g(u) \stackrel{\text { def }}{=} u-\frac{1}{2} \sin 2 u=\frac{2}{3} u^{3}+\mathcal{O}\left(u^{5}\right)
$$

We define

$$
Y(\tau)=e^{\tau / 2} R\left(T-e^{-\tau}\right)
$$

Then we have shown that

$$
Y(\tau) \leq C e^{-\tau / 2}
$$

for some constant $C<\infty$. 
Lemma 9.1. If one defines $U(\eta)=\pi-2 \arctan \eta$, then for all $y \geq Y(\tau)$ one has

$$
\left|u(y, \tau)-U\left(\frac{y}{Y(\tau)}\right)\right| \leq\left\{\begin{array}{rll}
C e^{-\tau / 2} y & \text { for } & y \geq Y(\tau) \\
C e^{-\tau / 2} & \text { for } & 0 \leq y \leq Y(\tau) .
\end{array}\right.
$$

Proof. Lemma 6.2 implies that

$$
|u(y, \tau)-U(y / Y(\tau))| \leq C e^{-X(\tau)}\left|\frac{y}{Y}-\frac{Y}{y}\right| \leq C e^{-\tau / 2} Y\left|\frac{y}{Y}-\frac{Y}{y}\right| .
$$

In the region $y \geq Y$ this directly implies the first inequality in (9.3).

For $0 \leq y \leq Y$ we get

$$
|u-U| \leq C e^{-\tau / 2} Y^{2} / y .
$$

In this region we also have $U \leq u \leq \pi$ so that

$$
|u-U| \leq \pi-U=2 \arctan y / Y \leq 2 y / Y .
$$

At each $y \in[0, Y]$ these two estimates imply that

$$
|u(y, \tau)-U(y / Y(\tau))| \leq \min \left\{2 y / Y, C e^{-\tau / 2} Y^{2} / y\right\} \leq C \sqrt{Y} e^{-\tau / 4} \leq C e^{-\tau / 2},
$$

since $\min \{a, b\} \leq \sqrt{a b}$ and in view of the estimate (9.2) for $Y(\tau)$.

We define

$$
m(\tau)=\int_{0}^{e^{\tau / 2}} y^{2} e^{-y^{2} / 4} u(y, \tau) d y .
$$

Lemma 9.2. One has

$$
m^{\prime}(\tau)=-\frac{1}{2} m(\tau)+(4+o(1)) Y(\tau)+\mathcal{O}\left(e^{-\frac{3}{2} \tau}\right) .
$$

Proof. One differentiates the defining equation (9.4) for $m(\tau)$ and obtains

$$
\begin{aligned}
m^{\prime}(\tau) & =e^{\tau} e^{-e^{\tau} / 4} u\left(e^{\tau / 2}, \tau\right)+\int_{0}^{e^{\tau / 2}} y^{2} e^{-y^{2} / 4} u_{\tau}(y, \tau) d y \\
& =\epsilon(\tau)+\int_{0}^{e^{\tau / 2}}\left\{y\left(y e^{-y^{2} / 4} u_{y}\right)_{y}-e^{-y^{2} / 4} u+e^{-y^{2} / 4} g(u)\right\} d y
\end{aligned}
$$

(integrate by parts twice)

$$
\begin{aligned}
& =\epsilon(\tau)-\frac{1}{2} \int_{0}^{e^{\tau / 2}} y^{2} e^{-y^{2} / 4} u(y, \tau) d \tau+\int_{0}^{e^{\tau / 2}} e^{-y^{2} / 4} g(u) d y \\
& =-\frac{1}{2} m(\tau)+\int_{0}^{e^{\tau / 2}} e^{-y^{2} / 4} g(u) d y+\epsilon(\tau) .
\end{aligned}
$$

Here $\epsilon(\tau)$ stands for a function of time which vanishes super exponentially, i.e., for some $C, c>0$ one has

$$
|\epsilon(\tau)| \leq C e^{-c e^{\tau}} .
$$


To complete the proof we must estimate the remaining integral. This is done in the following two propositions.

Proposition 9.3. $\int_{0}^{\infty} g(U(y)) d y=4$.

Proof. Since $U(y)$ satisfies the ODE $U^{\prime \prime}+\frac{1}{y} U^{\prime}=\frac{1}{2} y^{-2} \sin 2 U$, we have after integrating by parts a few times

$$
\begin{aligned}
\int_{0}^{L} g(U(y)) d y & =\int_{0}^{L}\left(-y^{2} U^{\prime \prime}(y)-y U^{\prime}(y)+U(y)\right) d y \\
& =\left[-y^{2} U^{\prime}(y)+y U(y)\right]_{y=0}^{y=L} \\
& =-L^{2} U^{\prime}(L)+L U(L) .
\end{aligned}
$$

For large $y$ we have $U(y) \sim 2 / y$ and hence $U^{\prime}(y) \sim-2 / y^{2}$. Taking the limit $L \rightarrow \infty$ in the above computation then proves the lemma.

Proposition 9.4.

$$
\int_{0}^{e^{\tau / 2}} g(u(y, \tau)) e^{-y^{2} / 4} d y=4 Y(\tau)+o(Y(\tau))+\mathcal{O}\left(e^{-(3 / 2) \tau}\right) .
$$

Proof. We split the integral into several pieces.

$$
\begin{aligned}
\int_{0}^{e^{\tau / 2}} g(u(y, \tau)) e^{-y^{2} / 4} d y= & \int_{0}^{e^{\tau / 2}} g(U(y / Y)) e^{-y^{2} / 4} d y \\
& \quad+\int_{0}^{e^{\tau / 2}}\{g(u(y, \tau))-g(U(y / Y))\} e^{-y^{2} / 4} d y \\
= & I_{1}+I_{2} .
\end{aligned}
$$

In the first integral we substitute $y=\eta Y$. The variable $\eta$ then runs from 0 to $e^{\tau / 2} / Y(\tau) \geq c e^{\tau}$. One finds

$$
\begin{aligned}
I_{1} & =Y(\tau) \int_{0}^{e^{\tau / 2} / Y(\tau)} g(U(\eta)) e^{-\eta^{2} Y^{2} / 4} d \eta \\
& =Y(\tau)(1+o(1)) \int_{0}^{\infty} g(U(\eta)) e^{-\eta^{2} Y^{2} / 4} d \eta \\
& =Y(\tau)(1+o(1)) \int_{0}^{\infty} g(U(\eta)) d \eta \\
& =(4+o(1)) Y(\tau)
\end{aligned}
$$

by Lemma 9.3 and monotone convergence.

In the second integral we use the mean value theorem, i.e., $g(u)-g(U)=g^{\prime}(\tilde{u})(u-$ $U$ ) for some $\tilde{u}$ between $u$ and $U$. Furthermore,

$$
0 \leq g^{\prime}(u)=1-\cos 2 u \leq C u^{2}
$$

for some constant, so we get $0 \leq g^{\prime}(\tilde{u}) \leq C\left(U^{2}+u^{2}\right)$. On the interval $Y \leq y \leq e^{\tau / 2}$ Lemma 9.1 tells us that

$$
U(y / Y(\tau))-C e^{-\tau / 2} y \leq u(y, \tau) \leq U(y / Y(\tau))
$$


so that $u^{2} \leq U^{2}+C e^{-\tau} y^{2}$. Applying this to the integral $I_{2}$ on the interval $Y \leq y \leq$ $e^{\tau / 2}$ we get

$$
\begin{aligned}
\mid \int_{Y}^{e^{\tau / 2}}\{g(u(y, \tau)) & -g(U(y / Y))\} e^{-y^{2} / 4} d y \mid \leq \\
& \leq C \int_{Y}^{e^{\tau / 2}}\left(U^{2}+u^{2}\right)|U-u| e^{-y^{2} / 4} d y \\
& \leq C e^{-\tau / 2} \int_{Y}^{e^{\tau / 2}}\left\{\left(\frac{Y}{y}\right)^{2}+e^{-\tau} y^{2}\right\} y e^{-y^{2} / 4} d y \\
& \leq C e^{-\tau / 2} Y(\tau)^{2} \ln \frac{1}{Y(\tau)}+C e^{-(3 / 2) \tau} \\
& \leq o(Y(\tau))+C e^{-(3 / 2) \tau} .
\end{aligned}
$$

For the integral from $y=0$ to $y=Y$ we have $|u-U|=\mathcal{O}\left(e^{-\tau / 2}\right)=o(1)$ uniformly, by Lemma 9.1. Since $g^{\prime}(u)$ is bounded, this implies

$$
\int_{0}^{Y(\tau)}|g(u(y, \tau))-g(U(y / Y))| e^{-y^{2} / 4} d y=o(Y(\tau)) .
$$

Adding the two pieces we get

$$
\left|I_{2}\right| \leq\left\{C e^{-\frac{3}{2} \tau}+o(Y(\tau))\right\} .
$$

The lemma is proved by adding the estimates for $I_{1}$ and $I_{2}$.

We can now improve our bound for the blow-up rates of $X(t)$ and $Y(\tau)$.

Proposition 9.5. For some constant $C<\infty$ one has $|m(\tau)| \geq C e^{-\tau / 2}$.

Proof. It follows from the estimates in Lemma 9.1 that $u(y, \tau) \geq-C y e^{-\tau / 2}$ for all $y$ and $\tau$. Substitution in (9.4) then gives $m(\tau) \geq-C e^{-\tau / 2}$.

To get the opposite inequality we recall Lemma 9.3, which says that for $y \geq Y$ one has $u(y, \tau) \leq U(y / Y)$, while for $y \in[0, Y]$ one has $u(y, \tau) \leq U(y / Y)+C e^{-\tau / 2}$. The explicit expression for $U(y / Y)$ implies that $U(y / Y) \leq 2 Y / y$. Substitution of these estimates in the definition of $m(\tau)$ gives $m(\tau) \leq C e^{-\tau / 2}$.

Proposition 9.6.

$$
\int_{\tau_{0}}^{\infty} e^{\tau / 2} Y(\tau) d \tau<\infty
$$

Proof. Apply the variation of constants formula to (9.5) to get

$$
e^{\tau / 2} m(\tau)-e^{\tau_{0} / 2} m\left(\tau_{0}\right)=\int_{\tau_{0}}^{\tau}\left\{(4+o(1)) e^{\sigma / 2} Y(\sigma)+\mathcal{O}\left(e^{-\sigma}\right)\right\} d \sigma .
$$

Since $Y(\tau)>0$ and since the left-hand side is bounded from above by the previous proposition, (9.6) follows.

Unraveling the definitions of $Y$ and $\tau$, we find

$$
\int_{0}^{T} R(t) \frac{d t}{(T-t)^{2}}<\infty
$$


To conclude we show how this integral bound also implies a pointwise bound. Recall that $R(t)$ is monotone, so that for any $\tau_{0}$ and $\tau \in\left(\tau_{0}-1, \tau_{0}\right]$ it follows from $Y(\tau)=e^{\tau / 2} R\left(T-e^{-\tau}\right)$ that

$$
Y(\tau)>e^{-1 / 2} Y\left(\tau_{0}\right)
$$

Hence

$$
\int_{\tau_{0}-1}^{\tau_{0}} e^{\tau / 2} Y(\tau) d \tau>e^{\tau_{0} / 2} e^{-1} Y\left(\tau_{0}\right)
$$

Convergence of the integral (9.6) then implies

$$
\lim _{\tau_{0} \rightarrow \infty} e^{\tau_{0} / 2} Y\left(\tau_{0}\right)=0
$$

which in turn implies $R(t)=o(T-t)$ as $t \nearrow T$.

\section{REFERENCES}

[1] M. Bertsch, R. D. PASso, And R. VAn DER Hout, Nonuniqueness for the heat flow of harmonic maps on the disk, Arch. Ration. Mech. Anal., 161 (2002), pp. 93-112.

[2] K.-C. Chang, W. Y. Ding, And R. Ye, Finite-time blow-up of the heat flow of harmonic maps from surfaces, J. Differential Geom., 36 (1992), pp. 507-515.

[3] J.-M. Coron And J.-M. Ghidaglia, Explosion en temps fini pour le flot des applications harmoniques, C. R. Acad. Sci. Paris Sér. I Math., 308 (1989), pp. 339-344.

[4] J. Eells, JR. And J. H. SAmpson, Harmonic mappings of Riemannian manifolds, Amer. J. Math., 86 (1964), pp. 109-160.

[5] L. D. Landau and E. Lifschitz, On the theory of the dispersion of magnetic permeability in ferromagnetic bodies, Phys. Z. Sowjet., 8 (1935), p. 153.

[6] M. Struwe, On the evolution of harmonic mappings of Riemannian surfaces, Comment. Math. Helv., 60 (1985), pp. 558-581.

[7] M. Struwe, Geometric evolution problems, in Nonlinear Partial Differential Equations in Differential Geometry, IAS/Park City Math. Ser. 2, Amer. Math. Soc., Providence, RI, 1996, pp. 257-339.

[8] M. Struwe, Variational methods, in Applications to Nonlinear Partial Differential Equations and Hamiltonian Systems, Ergeb. Math. Grenzgeb. (3) 34, Springer-Verlag, Berlin, 3rd ed., 2000.

[9] P. Topping, Repulsion and quantization in almost-harmonic maps, and asymptotics of the harmonic map flow, Ann. of Math. (2), 159 (2004), pp. 465-534.

[10] P. Topping, Winding behaviour of finite-time singularities of the harmonic map heat flow, Math. Z., 247 (2004), pp. 279-302.

[11] J. B. van Den Berg, J. Hulshof, And J. R. King, Formal asymptotics of bubbling in the harmonic map heat flow, SIAM J. Appl. Math., 63 (2003), pp. 1682-1717 (electronic).

[12] R. VAN DER Hout, Flow alignment in nematic liquid crystals in flows with cylindrical symmetry, Differ. Integral Equ., 14 (2001), pp. 189-211. 